\title{
Assistenten- und Assistentinnenumfrage 2003 aus Sicht der Leiter und Leiterinnen von Weiterbildungsstätten
}

\author{
Die wichtigsten Ergebnisse der Umfrage bei den Leitern und Leiterinnen von Weiterbildungsstätten
}

\author{
M. Siegrist ${ }^{a}$, M. Giger ${ }^{b}$
}

Im Frühjahr 2004 wurden die Leiter und Leiterinnen von 1402 anerkannten Weiterbildungsstätten zu den Resultaten der Assistentenumfrage 2003, zu ihrer Wahrnehmung der Assistenzärztinnen und Assistenzärzte sowie zur Bedeutung der Weiterbildung befragt. Der Grossteil der Befragten fühlt sich korrekt beurteilt. Die Resultate der Assistentenumfrage 2003 lösten bei rund zwei Dritteln der antwortenden Leiter und Leiterinnen Massnahmen zur Verbesserung der Weiterbildung aus. Die Assistenten werden im Vergleich zu früher von $57 \%$ als weniger einsatzfreudig wahrgenommen. Von der Hälfte wird ihnen zuwenig Eigeninitiative im Bereiche der Weiterbildung attestiert. Neun von zehn antwortenden Leiter und Leiterinnen möchten sich auch in Zukunft in der Weiterbildung engagieren.

\footnotetext{
a Sozialforschungsstelle der Universität Zürich

b Ressort Medical Education FMH
}

Korrespondenz: PD Dr. phil. Michael Siegrist Universität Zürich Sozialpsychologie Plattenstrasse 14 CH-8032 Zürich
Die eigenen Leistungen objektiv zu beurteilen, fällt vielen schwer. Wir stufen uns positiver ein als andere Menschen [1]. Auch unsere eigenen Fähigkeiten sehen wir in einem rosa Licht. So waren in einer Untersuchung deutlich über 50\% der Meinung, überdurchschnittlich gute Autofahrer zu sein [2]. Diese Überschätzung der eigenen Fähigkeiten und Leistungen beschränkt sich natürlich nicht auf das Autofahren, sondern konnte in zahlreichen anderen Bereichen ebenfalls beobachtet werden.

Vorgesetzte und Weiterbildner erhalten im Alltag nur in ungenügendem Ausmass kritische Rückmeldungen. Zu den Gründen zählen die Bequemlichkeit und möglicherweise auch Konfliktscheu der Mitarbeiter. Aber auch die Asymmetrie zwischen Weiterbildner und Assistenzarzt dürfte eine Rolle spielen. Die Abhängigkeit der Assistenzärzte begünstigt eine offene, kritische Rückmeldung nicht. Der Weiterbildner braucht aber ehrliche Rückmeldungen, damit die eigenen Leistungen richtig eingeschätzt werden. Die Literatur zeigt zudem, dass Rückmeldungen in vielen Fällen einen positiven Einfluss auf die Leistung haben [3]. Aus diesen Gründen erscheint eine institutionalisierte Feedbackkultur ein wichtiges Element einer qualitätsorientierten Weiterbildung zu sein.
Für die Umfrage 2003 bei den Assistenzärzten und Assistenzärztinnen zur Beurteilung der Weiterbildung wurde ein vollständig neuer Fragebogen erarbeitet $[4,5]$. Dieser Fragebogen ermöglicht relativ detaillierte Rückmeldungen an die Leiter und Leiterinnen der Weiterbildungsstätten. Wir haben zahlreiche individuelle Rückmeldungen zur Umfrage 2003 erhalten - sowohl positive als auch negative. Wir wollten aber ein umfassendes Bild darüber erhalten, wie die Umfrage 2003 von den Leitern und Leiterinnen von Weiterbildungsstätten beurteilt wird. Deshalb wurde eine systematische Befragung durchgeführt. Zusätzlich wurden auch Fragen ganz allgemein zur Weiterbildung und zur Wahrnehmung der Assistenzärztinnen und Assistenzärzte gestellt. Zum ersten Mal liegen aussagekräftige Daten vor, die zeigen, wie die Verantwortlichen die Weiterbildung in den Schweizer Spitälern beurteilen.

\section{Methode}

\section{Stichprobe}

Im Rahmen der statistischen Erhebung bei den Leitern und Leiterinnen der Weiterbildungsstätten wurden inhaltliche Fragen zu unterschiedlichen Aspekten der Weiterbildung gestellt. Die Befragung erfolgte im Frühling 2004 auf elektronischem Weg bei 1402 von der FMH anerkannten Weiterbildungsstätten. Ein Teil der Fragen richtete sich an alle Befragten, ein Teil der Fragen war nur von den Befragten zu beantworten, die den Bericht 2003 erhalten hatten. Bei den Fragen, welche von allen beantwortet werden konnten, lag die Antwortquote zwischen 92\% $(\mathrm{N}=1289)$ und $79 \%(\mathrm{~N}=1111)$. Die Befragten wurden nach ihrer Funktion innerhalb der Institution befragt. Von den Teilnehmenden gaben 80,6\% Chefarzt, 14,0\% leitender Arzt, 1,4\% Oberarzt und $3,6 \%$ anderes an. 


\section{Fragebogen}

Der Fragebogen enthielt 20 Fragen. In erster Linie wurden die Befragten mit Aussagen konfrontiert. Für diese Aussagen standen sechs Antwortkategorien zur Auswahl, von 1 «trifft überhaupt nicht zu» bis 6 «trifft voll und ganz zu». Die Zahlen dazwischen waren nicht verbal verankert.

\section{Ergebnisse}

\section{Beurteilung der Umfrage 2003}

Den Leiterinnen und Leitern von Weiterbildungsstätten, welche 2003 eine Rückmeldung der Umfrage zur Beurteilung der Weiterbildung durch Assistenzärzte und Assistenzärztinnen erhalten hatten, wurden spezifische Fragen dazu gestellt. Für 980 Weiterbildungsstätten konnte ein Bericht erstellt werden. Die Befragten konnten angeben, welche Aktivitäten durch den Bericht ausgelöst wurden. Bei 788 (80\%) Weiterbildungsstätten wurden die Ergebnisse mit Oberärzten/leitenden Ärzten besprochen. Bei 680 (69\%) Weiterbildungsstätten wurden die Ergebnisse mit Assistenzärzten besprochen, und bei 615 (63\%) Weiterbildungsstätten wurden konkrete Schritte für die Verbesserung der Weiterbildungsqualität eingeleitet.

In Tabelle 1 sind die Antworten zu den weiteren Fragen zum Bericht 2003 aufgeführt. 48\% der Befragten gaben an, etwas Neues über die Weiterbildungsstätten erfahren $\mathrm{zu}$ haben. Ein grosser Teil der Befragten fühlt sich durch die Umfrage gerecht beurteilt. Nur jeder vierte Be- fragte hatte das Gefühl, mehr oder weniger ungerecht beurteilt worden zu sein. Von den Befragten gaben $66 \%$ an, dass die Ergebnisse der Umfrage Anlass waren, die Weiterbildung zu überdenken, und $63 \%$ gaben an, dass konkrete Hinweise aus der Umfrage Anlass waren, Veränderungsprozesse einzuleiten.

\section{Wie werden Assistenzärzte und -ärztinnen wahrgenommen?}

Tabelle 2 kann entnommen werden, wie die Befragten die Assistenzärzte wahrnehmen. Die Assistenzärzte und Assistenzärztinnen von heute werden von 57\% der Antwortenden als weniger einsatzfreudig wahrgenommen als die von früher. Die Hälfte stellt zudem eine mangelnde Eigeninitiative der Assistenzärzte im Bereich der Weiterbildung fest. Immerhin 41\% sind der Ansicht, dass die Assistenzärzte und Assistenzärztinnen von heute schwieriger zu motivieren sind als früher. Jeder zweite vertritt die Ansicht, dass für die Weiterbildung kaum zusätzliche Stunden geleistet werden, welche die gesetzlich festgelegten Arbeitszeiten übersteigen. Erfreulich ist, dass 85\% der Antwortenden der Meinung sind, dass die Assistenzärzte ihre Arbeit an den meisten Tagen mit Begeisterung ausüben. Den Assistenzärzten und Assistenzärztinnen wird also eine relativ hohe Motivation zugeschrieben.

Die Antworten zur Beurteilung der Eigeninitiative im Bereiche der Weiterbildung für die vier Fachgebiete mit den meisten Weiterbildungsstätten können Tabelle 3 entnommen werden. Bei den Befragten aus dem Bereich Psychiatrie und Psychotherapie gab es die geringste

Tabelle 1

Zeilenprozente für vier Fragen zum individuellen Bericht 2003, welcher für die Leiter und Leiterinnen von Weiterbildungsstätten erstellt worden war.

\begin{tabular}{|c|c|c|c|c|c|}
\hline $\begin{array}{l}1 \text { (trifft überhaupt } \\
\text { nicht zu) }\end{array}$ & 2 & 3 & 4 & 5 & $\begin{array}{l}6 \text { (trifft voll } \\
\text { und ganz zu) }\end{array}$ \\
\hline \multicolumn{6}{|c|}{ Durch den Bericht habe ich etwas Neues über unsere Weiterbildungsstätte erfahren. } \\
\hline $13,0 \%$ & $19,6 \%$ & $19,7 \%$ & $23,3 \%$ & $18,7 \%$ & $5,7 \%$ \\
\hline \multicolumn{6}{|c|}{ Unsere Weiterbildungsstätte wurde in der Umfrage gerecht beurteilt. } \\
\hline $4,3 \%$ & $8,8 \%$ & $12,0 \%$ & $19,8 \%$ & $36,0 \%$ & $19,1 \%$ \\
\hline \multicolumn{6}{|c|}{ Die Resultate der Umfrage waren Anlass für mich, unsere Weiterbildung zu überdenken. } \\
\hline $7,8 \%$ & $13,7 \%$ & $13,0 \%$ & $23,9 \%$ & $30,7 \%$ & $10,9 \%$ \\
\hline \multicolumn{6}{|c|}{ Die konkreten Hinweise aus der Umfrage haben mich motiviert, Veränderungsprozesse einzuleiten. } \\
\hline $7,9 \%$ & $13,9 \%$ & $15,2 \%$ & $27,2 \%$ & $27,2 \%$ & $8,7 \%$ \\
\hline
\end{tabular}


Tabelle 2

Zeilenprozente für fünf Fragen zur Wahrnehmung der Assistenzärzte und -ärztinnen.

\begin{tabular}{|c|c|c|c|c|c|}
\hline $\begin{array}{l}1 \text { (trifft überhaupt } \\
\text { nicht } \mathrm{zu} \text { ) }\end{array}$ & 2 & 3 & 4 & 5 & $\begin{array}{l}6 \text { (trifft voll } \\
\text { und ganz zu) }\end{array}$ \\
\hline \multicolumn{6}{|c|}{ Die heutigen Assistenzärzte sind nicht mehr so einsatzfreudig wie früher. } \\
\hline $12,6 \%$ & $19,7 \%$ & $10,6 \%$ & $19,3 \%$ & $22,4 \%$ & $15,5 \%$ \\
\hline \multicolumn{6}{|c|}{ Unsere Assistenzärzte zeigen zuwenig Eigeninitiative im Bereich der Weiterbildung. } \\
\hline $9,9 \%$ & $23,7 \%$ & $16,4 \%$ & $22,8 \%$ & $21,0 \%$ & $6,2 \%$ \\
\hline \multicolumn{6}{|c|}{ Die Assistenzärzte von heute sind schwieriger zu motivieren als früher. } \\
\hline $14,4 \%$ & $27,7 \%$ & $16,5 \%$ & $19,3 \%$ & $16,1 \%$ & $5,9 \%$ \\
\hline \multicolumn{6}{|c|}{$\begin{array}{l}\text { Die Assistenzärzte halten sich streng an die vertraglich festgelegten Arbeitszeiten. Für die Weiterbildung } \\
\text { werden kaum zusätzliche Stunden geleistet. }\end{array}$} \\
\hline $11,5 \%$ & $21,4 \%$ & $16,7 \%$ & $21,0 \%$ & $22,0 \%$ & $7,4 \%$ \\
\hline \multicolumn{6}{|c|}{ Die Assistenzärzte an meiner Weiterbildungsstätte üben ihre Arbeit an den meisten Tagen mit Begeisterung aus. } \\
\hline $3,3 \%$ & $4,9 \%$ & $7,0 \%$ & $22,7 \%$ & $46,2 \%$ & $15,9 \%$ \\
\hline
\end{tabular}

Tabelle 3

Antworten und Kolonnenprozente für die Aussage «Unsere Assistenzärzte zeigen zuwenig Eigeninitiative im Bereich der Weiterbildung» für die vier Fachrichtungen mit den meisten Weiterbildungsstätten.

\begin{tabular}{|c|c|c|c|c|}
\hline & Innere Medizin & Chirurgie & $\begin{array}{l}\text { Gynäkologie } \\
\text { und Geburtshilfe }\end{array}$ & $\begin{array}{l}\text { Psychiatrie } \\
\text { und Psychotherapie }\end{array}$ \\
\hline Ablehnend & $44,5 \%(81)$ & $33,3 \%(37)$ & $48,1 \%(37)$ & $59,8 \%(64)$ \\
\hline Zustimmend & $55,5 \%(101)$ & $66,7 \%(74)$ & $51,9 \%(40)$ & $40,2 \%(43)$ \\
\hline Total & $100 \%(182)$ & $100 \%(111)$ & $100 \%(77)$ & $100 \%(107)$ \\
\hline \multicolumn{5}{|c|}{$\chi_{(3)}^{2}=15,69, p=0,001$} \\
\hline
\end{tabular}

Tabelle 4

Zeilenprozente für drei Fragen zur Zukunft der Weiterbildung.

\begin{tabular}{|c|c|c|c|c|c|}
\hline $\begin{array}{l}1 \text { (trifft überhaupt } \\
\text { nicht zu) }\end{array}$ & 2 & 3 & 4 & 5 & $\begin{array}{l}6 \text { (trifft voll } \\
\text { und ganz zu) }\end{array}$ \\
\hline \multicolumn{6}{|c|}{ Am liebsten würde ich auf die Weiterbildung von Assistenzärzten (in Weiterbildung) verzichten. } \\
\hline $61,4 \%$ & $21,4 \%$ & $5,9 \%$ & $6,9 \%$ & $3,2 \%$ & $1,2 \%$ \\
\hline \multicolumn{6}{|c|}{ In absehbarer Zeit wird es an unserem Spital wohl keine Assistenzärzte (in Weiterbildung) mehr geben. } \\
\hline $51,1 \%$ & $21,9 \%$ & $10,6 \%$ & $7,9 \%$ & $5,4 \%$ & $3,1 \%$ \\
\hline \multicolumn{6}{|c|}{$\begin{array}{l}\text { Mit Spitalärzten könnte meine Abteilung in mindestens gleicher Qualität und kostengünstiger arbeiten als mit Assistenz- } \\
\text { ärzten (in Weiterbildung). }\end{array}$} \\
\hline $17,7 \%$ & $17,3 \%$ & $18,0 \%$ & $16,5 \%$ & $19,4 \%$ & $11,1 \%$ \\
\hline
\end{tabular}


Tabelle 5

Antworten und Kolonnenprozente für die Aussage «In absehbarer Zeit wird es an unserem Spital wohl keine Assistenzärzte (in Weiterbildung) mehr geben» für die vier Fachrichtungen mit den meisten Weiterbildungsstätten.

\begin{tabular}{|c|c|c|c|c|}
\hline & Innere Medizin & Chirurgie & $\begin{array}{l}\text { Gynäkologie } \\
\text { und Geburtshilfe }\end{array}$ & $\begin{array}{l}\text { Psychiatrie } \\
\text { und Psychotherapie }\end{array}$ \\
\hline Ablehnend & $85,3 \%(157)$ & $71,3 \%(77)$ & $81,8 \%(63)$ & $92,3 \%(96)$ \\
\hline Zustimmend & $14,7 \%(27)$ & $28,7 \%(31)$ & $18,2 \%(14)$ & $7,7 \%(8)$ \\
\hline Total & $100 \%(184)$ & $100 \%(108)$ & $100 \%(77)$ & $100 \%(104)$ \\
\hline \multicolumn{5}{|c|}{$\chi^{2}(3)=17,72, p=0,001$} \\
\hline
\end{tabular}

Zustimmung zur Aussage «Unsere Assistenzärzte zeigen zuwenig Eigeninitiative im Bereich der Weiterbildung». In der Chirurgie wurde diese Aussage dagegen nur von jeder dritten befragten Person abgelehnt.

\section{Wie wird die Zukunft der Weiterbildung eingeschätzt?}

Weiterbildung ist für einen grossen Teil der Befragten wichtig (Tab. 4). Nur 11\% der Antwortenden stimmten der Aussage «Am liebsten würde ich auf die Weiterbildung von Assistenzärzten (in Weiterbildung) verzichten» mehr oder weniger zu. Die meisten der Befragten gehen auch davon aus, dass in ihrem Spital weiterhin Assistenzärzte weitergebildet werden. Jeder zweite Befragte stimmte der Ansicht mehr oder weniger zu, dass mit Spitalärzten in mindestens gleicher Qualität und kostengünstiger gearbeitet werden könnte.

Die Fachrichtung hat einen signifikanten Einfluss darauf, wie die künftige Einbettung der Weiterbildung in die heutigen Weiterbildungsstätten gesehen wird (Tab. 5). Bei den Chirurgen stimmten weit mehr Befragte der Ansicht mehr oder weniger zu, dass es in absehbarer Zeit am eigenen Spital keine Assistenzärzte mehr geben werde, als Befragte anderer Fachrichtungen.

\section{Diskussion}

Die Rücklaufquote dieser Umfrage bei den Leiterinnen und Leitern der Weiterbildungsstätten liegt mit rund $80 \%$ deutlich über derjenigen bei den Assistenzärztinnen und Assistenzärzten [5]. Der Grossteil der Leiterinnen und Leiter von Weiterbildungsstätten fühlt sich durch die Assistentenumfrage 2003 korrekt beurteilt.

Ein erfreulich grosser Teil der Befragten gibt an, aufgrund der Umfrage die Weiterbildung hinterfragt oder gar Veränderungsprozesse eingeleitet zu haben. Das Ziel, mit der Umfrage den Leitern und Leiterinnen möglichst konkrete Hinweise auf Veränderungspotentiale zu geben, wurde also zu einem grossen Grad erreicht.

Weil für alle Fachgesellschaften der identische Fragebogen verwendet wurde, müssen die Fragen einen gewissen Abstraktionsgrad haben, sonst können sie nicht von allen Assistenzärzten und Assistenzärztinnen beantwortet werden. Bei künftigen Erhebungen sollten diese allgemeinen Teile des Fragebogens durch fachspezifische Fragen ergänzt werden - zumindest bei den Fachrichtungen mit den meisten Assistenzärzten und Assistenzärztinnen. Mehrere Fachgesellschaften arbeiten solche spezifischen Fragen aus, welche bei der Umfrage im nächsten Jahr berücksichtigt werden sollen. Dadurch lässt sich differenzierter überprüfen, wie gut die Weiterbildungsziele in einer bestimmten Fachrichtung erreicht werden.

Bei der Wahrnehmung der Assistenzärzte und Assistenzärztinnen kann eine gewisse Polarisierung beobachtet werden. Ungefähr die Hälfte der Befragten nimmt die heutigen Assistenzärzte und Assistenzärztinnen als weniger einsatzfreudig und schwieriger $\mathrm{zu}$ motivieren wahr, als dies früher der Fall war. Ebenfalls etwa die Hälfte der Befragten ist der Meinung, dass die Assistenzärzte und Assistenzärztinnen bei der Weiterbildung zuwenig Initiative zeigen und dafür kaum zusätzliche Stunden leisten. Trotz dieser kritischen Einschätzung der heutigen Assistenzärzte und Assistenzärztinnen wird ihnen eine grosse Motivation zugeschrieben. Auch scheint für einen grossen Teil der Befragten die Weiterbildung wichtig zu sein, etwas, auf das sie nicht verzichten möchten.

Die signifikant unterschiedliche Beurteilung der Eigeninitiative der Assistenten im Bereiche der Weiterbildung durch die Leiter der psychiatrischen und chirurgischen Weiterbildungs- 
stätten ist höchstwahrscheinlich auf den Anteil der fachfremden Assistenten bzw. eine geringere Zufriedenheit der Assistenten zurückzuführen [5].

Dass ein grosser Teil der Befragten sich hinter die Weiterbildung stellt, darf als positives Zeichen für die Zukunft gewertet werden. Die prognostizierte Reduktion von Weiterbildungsstellen vor allem in der Chirurgie bedarf einer tieferen Analyse, um Qualität und Quantität der Weiterbildung in diesem Gebiet nicht zu gefährden.

Strukturierte Rückmeldungen sind ein Instrument zur Verbesserung der Weiterbildungsqualität. Dieses Ziel wird aber nur dann erreicht, wenn die Rückmeldungen von den meisten Betroffenen als fair und akzeptabel eingestuft werden. Beides scheint auf die Umfrage 2003 zuzutreffen. Auch wenn der Fragebogen 2003, wie die vorliegenden Resultate zeigen, insgesamt recht positiv beurteilt wurde, wurde dieser für die Umfrage 2004 weiter verbessert. Dabei wurde versucht, die Wünsche der Betroffenen soweit wie möglich zu berücksichtigen. Da es bei den zahlreichen Anregungen und Verbesserungsvor- schlägen aber auch Zielkonflikte gab, konnten nicht alle Rückmeldungen berücksichtigt werden.

\section{Literatur}

1 Alicke MD, Klotz ML, Breitenbecher DL, Yurak TJ, Vredenburg DS. Personal contact, individuation, and the better-than-average effect. Journal of Personality and Social Psychology 1995;68:804-25.

2 Svenson O. Are we all less risky and more skillful than our fellow drivers? Acta Psychologica 1981; 47:143-8.

3 Kluber AN, DeNisi A. The effects of feedback interventions on performance: A historical review, a meta-analysis, and a preliminary feedback intervention theory. Psychological Bulletin 1996; 119:254-84.

4 Siegrist M, Giger M. Ärztliche Weiterbildung auf dem Prüfstand. Schweiz Ärztezeitung 2003; 84(50):2655-7.

5 Siegrist M, Gutscher H, Giger M. Was haben Betriebskultur, Motivation und Fremdjahr mit der Weiterbildung zu tun? Schweiz Ärztezeitung 2004;85(15):783-91. 